\title{
Liver Metastases and Peritoneal Carcinomatosis in A Sigmoid Adenocarcinoma Should We Perform Colectomy in A Stage IV Colorectal Cancer?
}

\section{Beatriz Losada*, María del Carmen Pantín, Javier de la Rubia, Israel Manzanedo and David Gutiérrez}

University Hospital of Fuenlabrada, Madrid, Spain

"Corresponding author: Losada B, University Hospital of Fuenlabrada, Madrid, Spain, Tel: 677098761; E-mail: beatriz.losada@salud.madrid.org

Received date: December 06, 2017; Accepted date: December 14, 2017; Published date: December 21, 2017

Copyright: @ 2017 Losada B, et al. This is an open-access article distributed under the terms of the Creative Commons Attribution License, which permits unrestricted use, distribution, and reproduction in any medium, provided the original author and source are credited.

\section{Abstract}

Colorectal cancer is the second cause of death in Spain (20\% diagnosed in stage IV), improving survival in recent years thanks to chemotherapy and metastatic surgeries.

With regard to a patient diagnosed with native stage IV KRAS colon adenocarcinoma (peritoneal, hepatic), the subject of cytoreduction with HIPEC (intraperitoneal hyperthermic chemotherapy) and the factors that influence the tumor response are reviewed.
\end{abstract}

\section{Introduction}

Colorectal cancer is the second cause of death in Spain, despite improving local and systemic treatment (nowadays metastatic colorectal cancer has achieved a 5 -year survival of 50\%). However not all the patients can receive these treatment, so we have to improve and select those who can benefit from antiangiogenics and hypertermic peritoneal cytoreduction (HIPEC). Therefore, it is very important to decide the best sequence of treatment in a Multidisciplinary Tumor Committee and to know more about molecular factors to help us to choose between different chemotherapy agents, so investigation and clinical trials are key.

\section{Clinical Case}

A 57 years old woman with no personal history to note went to the Emergency Department in October 2016 due to abdominal pain of a week of evolution. Physical exploration showed abdomen distended, without hydro-aerial noise and signs of peritoneal irritation. We performed a CT (26-10-2016) which showed a extensive peritoneal carcinomatosis, ascites and possible liver metastases. Intestinal perforation, probably related to tumor in the left colon (Figure 1).

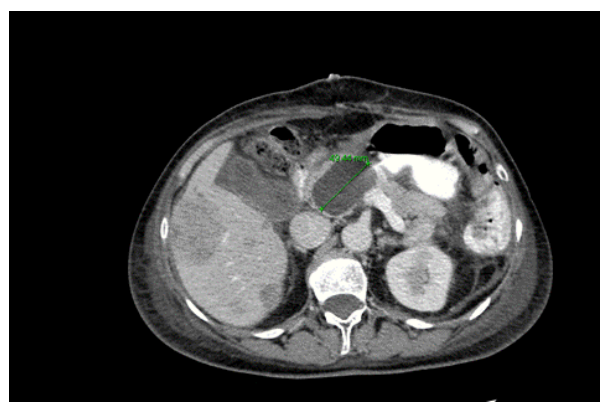

Figure 1: Liver metastases in the diagnosis.
With these findings, differential diagnosis is between:

- Peritoneal carcinomatosis secondary to digestive/gynecological tumor process.

- Hypodense liver lobes, suggestive of metastases of colorectal origin (not hypervascular, so it does not suggest neuroendocrine origin).

Afterwards, urgent surgical intervention was decided in which extensive carcinomatosis with estimated Peritoneal Carcinomatosis Index (PCI) of 32 points was found, as well as 2 liver LOEs greater than $5 \mathrm{~cm}$ in segments VII and VIII.

${ }^{*}$ Colectomy: Adenocarcinoma of colorectal origin pT4b N2b (22/24) M1b L1V1Pn1 KRAS/NRAS/BRAF negative.

Given the extensive unresectable tumor disease, chemotherapy is initiated with palliative intent (FOLFOX+Cetuximab) on day 18-11-16. Good tolerance, highlighting only skin rash grade 1. It covers 4 cycles until 04.01.17 with improved levels of markers tumors (Graph 1) and partial tumor response on CT ( $>50 \%$ in size of liver metastases and peritoneal implants).

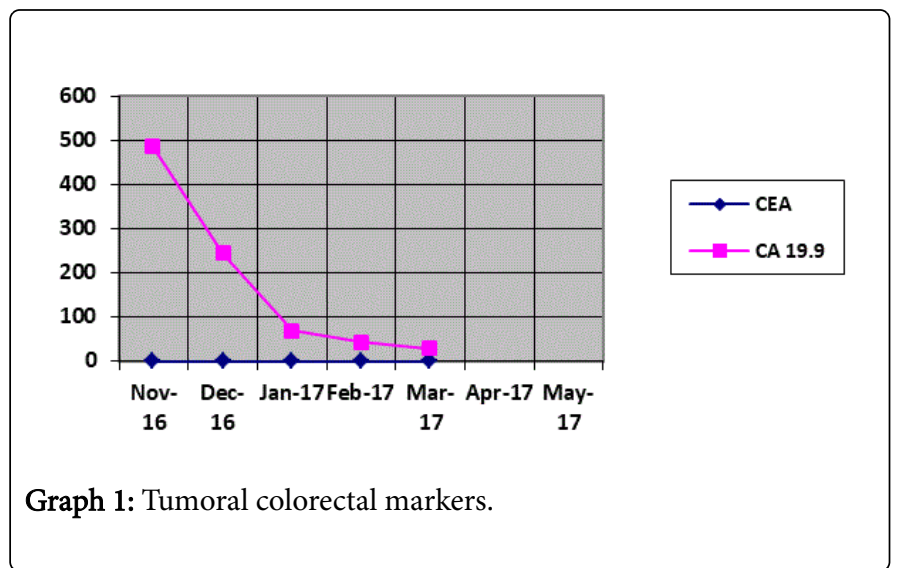

The same chemotherapy scheme continues, but in the $8^{\text {th }}$ cycle (March 2017) there is a worsening of rash to grade 2-3 in the 
Citation: Losada B, del Carmen Pantín M, de la Rubia J, Manzanedo I, Gutiérrez D (2017) Liver Metastases and Peritoneal Carcinomatosis in A Sigmoid Adenocarcinoma Should We Perform Colectomy in A Stage IV Colorectal Cancer? J Oncol Transl Res 3: 124. doi: $10.4172 / 2476-2261.1000124$

Page 2 of 2

periorbital area and grade 2 transaminitis. Due to dermatology toxicity, doxycycline is initiated.

Restarts treatment with dose reduction of $20 \%$ of cetuximab and oxaliplatin. Partial response on CT (03-05-17, Figure 2) so it is presented in Multidisciplinar Committee of Carcinomatosis and cytoreduction is decided (28-06-17) as well as limited resection of segment $5+$ Radiofrequency segments 5 and $6+$ left nephrectomy + Hyperthermic Chemotherapy with cytoreduction. Initial PCI was of 8 points and the degree of cytoreduction $=0$.

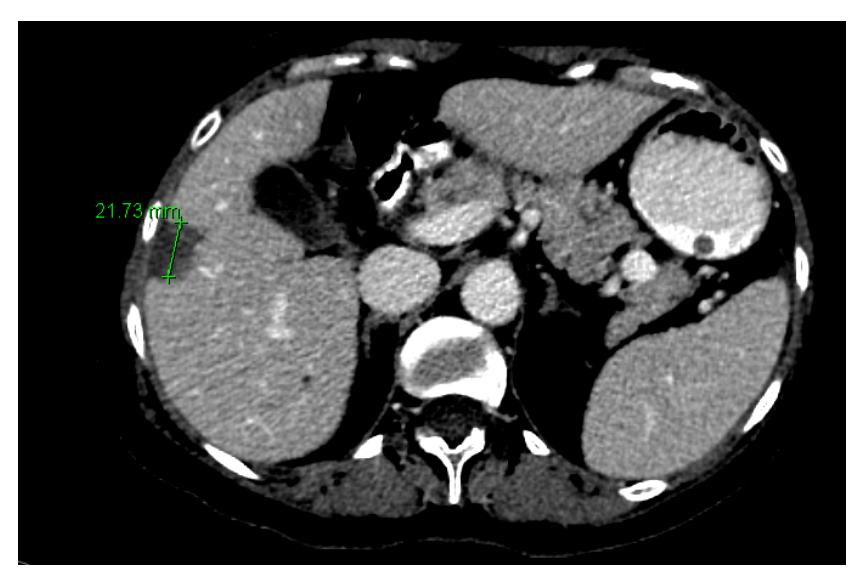

Figure 2: Partial liver response.

Postoperatory without alterations, so he continues "adjuvant chemo" with FOLFIRI+Cetuximab without presenting tumor disease after 12 months of the diagnosis of a stage IV colon adenocarcinoma.

\section{Discussion}

Liver resectability criteria consist in guaranteeing an R0 resection, preserving 2 contiguous hepatic segments and that the hepatic remnant can be functionally adequate [1].

Regarding peritoneal carcinomatosis, it is true that $\mathrm{PCI}>20$ confers a worse prognosis as well as the presence of visceral (hepatic) metastases. However, recently it has been published that if the patient has $<3$ liver metastases as well as a $\mathrm{PCI}<12$ or good response after chemotherapy, the surgical decision must be individualized [2].
FOLFOX+Cetuximab (OPUS study) is indicated in potentially resectable native KRAS colon cancer [3]. Imaging technique of choice for hepatic resectability assessment is both MRI and PET-CT (for extrahepatic involvement) [4].

Anti-EGFR can redirect the immune response against tumor cells by interacting with $\mathrm{CD} 3 / \mathrm{CD} 16$ expression and exacerbating characteristic side effects such as rash, being a predictor of response [5].

\section{Conclusion}

Metastatic colon cancer has achieved a 5 years survival of $50 \%$ thanks to the individualization of surgical criteria and availability of targeted treatments. This case includes as novelty the fact of adding HIPEC to metastatic colorectal cancer with a high PCI score and the possible inmune relation of an EGFR.

A high PCI index should guide us towards a worse prognosis but the surgical decision must be decided in a Multidisciplinary Tumor Committee, so this can be useful for other patients in this same situation.

Every time we know more molecular factors (KRAS, BRAF, PI3K) as well as clinical signs such as the rash with predictive value of response.

\section{References}

1. Charnsangavej C, Clary B, Fong Y, Grothey A, Pawlik TM, et al. (2006) Selection of patients for resection of hepatic colorectal metastases: Expert consensus statement. Ann Surg Oncol 13: 1261-1268.

2. Mo S, Cai G (2016) Multidisciplinary treatment for colorectal peritoneal metastases: Review of the literature. Gastroenterol Res Pract 6: 1516259.

3. Bokemeyer C, Bondarenko I, Hartmann JT, de Braud F, Schuch G, et al. (2011) Efficacy according to biomarker status of cetuximab plus FOLFOX-4 as first-line treatment for metastatic colorectal cancer: The OPUS study. Ann Oncol 22: 1535-1546.

4. Legou F, Chiaradia M, Baranes L, Pigneur F, Zegai B, et al. (2014) Imaging strategies before beginning treatment of colorectal liver metastases. Diagn Interv Imaging 95: 505-512.

5. Snyder LC, Astsaturov I, Weiner LM (2005) Overview of monoclonal antibodies and small molecules targeting the epidermal growth factor receptor pathway in colorectal cancer. Clin Colorectal Cancer 5: S71-S80. 